\title{
REVIEW
}

\section{A Review of the Key Clinical Trials of 2014}

\author{
Peter McKavanagh • Claire McCune $\cdot$ Ian B. Menown
}

To view enhanced content go to www.cardiologytherapy-open.com

Received: January 23, 2015 / Published online: March 27, 2015

(c) The Author(s) 2015. This article is published with open access at Springerlink.com

\section{ABSTRACT}

Introduction: Over the last year, multiple, potentially practice-changing, cardiology trials or studies have been published or presented at international meetings including the American College of Cardiology, European Association for Percutaneous Cardiovascular Interventions, European Society of Cardiology, Transcatheter Cardiovascular Therapeutics, Heart Failure Congress, Heart Rhythm Society, Heart Failure Society of America, American Society of Hypertension and the American Heart Association.

Methods: Clinical trial results presented at major cardiology conferences during 2014 were reviewed by the authors. Search terms included heart failure (HF), acute coronary syndrome, stable coronary disease, interventional cardiology, atrial fibrillation, electrophysiology and coronary prevention.

Electronic supplementary material The online version of this article (doi:10.1007/s40119-015-0036-0) contains supplementary material, which is available to authorized users.

P. McKavanagh ( $)$ · C. McCune · I. B. Menown Craigavon Cardiac Centre, Southern Trust, Craigavon BT63 5QQ, Northern Ireland, UK e-mail: mckavanagh@doctors.org.uk
Selection criteria were trials of broad relevance to the cardiology community, those with potential to change current practice and those with potential to guide further phase III research.

Results: In this paper, the authors describe and place in clinical context, new HF, data including neprilysin inhibitors, intravenous ferric carboxymaltose, potassium-absorbing compounds, quadripolar leads for cardiac resynchronization therapy and intraventricular device intervention. New trial data are also described for acute coronary syndromes (clopidogrel, prasugrel, ticagrelor), stable coronary artery disease (ivabradine), percutaneous coronary intervention (the role of thrombectomy or treatment of non-culprit lesions during primary intervention, pressure wire studies and outcomes of new stent designs), transcatheter aortic valve intervention data, atrial fibrillation (anticoagulation and direct current cardioversion), electrophysiology (leadless pacemaker devices, use of quinidine in Brugada syndrome) and coronary prevention (landmark Ezetimibe outcome data, PCSK9 clinical trials, childhood prevalence of 
hypertension, renal denervation for resistant hypertension and the role of cardiac computerized tomography in cardiovascular screening).

Conclusion: This paper summarizes key clinical trials during 2014 and should be of practical interest to clinicians and cardiology researchers.

Keywords: Acute coronary syndromes; Anticoagulation; Antiplatelet; Atrial fibrillation; Bioabsorbable polymer; Cardiology; Heart failure; Myocardial infarction; Pacemaker; Revascularization

\section{INTRODUCTION}

Cardiovascular (CV) medicine is a specialty defined by research milestones [1]. This strong research ethos and abundance of emerging research can make it difficult for the clinical cardiologist to keep up to date with the latest evidence. Throughout 2014, a number of breaking trials have been presented at major international cardiology conferences with the potential to impact guidelines and practice. In this paper, we describe and place in clinical context new data for heart failure (HF), acute coronary syndrome, stable coronary disease, interventional cardiology, atrial fibrillation, electrophysiology and coronary prevention.

\section{METHODS}

Clinical trial results presented at major cardiology conferences during 2014 were reviewed by the authors. Conferences included the American College of Cardiology (ACC), European Association for Percutaneous Cardiovascular Interventions (EuroPCR), European Society of Cardiology (ESC), Transcatheter Cardiovascular Therapeutics
(TCT), Heart Failure Congress, Heart Rhythm Society (HRS), Heart Failure Society of America (HFSA), American Society of Hypertension (ASH), Society for Cardiovascular Angiography Interventions (SCAI) and the American Heart Association (AHA). Search terms included heart failure, acute coronary syndrome, stable coronary disease, interventional cardiology, atrial fibrillation, electrophysiology and coronary prevention. Selection criteria were trials of broad relevance to the cardiology community, those with potential to change current practice and those with potential to guide further phase III research. This article is based on previously conducted studies and does not involve any new studies of human or animal subjects performed by any of the authors.

\section{DISCUSSION}

\section{Heart Failure}

For the past two decades, angiotensinconverting enzyme (ACE) inhibitors have been considered the cornerstone of HF therapy. Neprilysin is a neutral endopeptidase which degrades potentially beneficial natriuretic and vasoactive peptides. The prospective comparison of ARNI with ACEI to Determine Impact on Global Mortality and Morbidity in HF (PARADIGM-HF) study (ClinicalTrials.gov \#NCT01035255) randomized 8442 patients with reduced left ventricular (LV) ejection fraction and New York Heart Association (NYHA) class II-III symptoms to LCZ696 (a combination of the neprilysin inhibitor sacubitril and valsartan) or to standard treatment with enalapril [2]. The trial was stopped early after a mean follow-up of 27 months, at which point LCZ696 compared 
with enalapril resulted in a $20 \%$ decrease in primary end point of $\mathrm{CV}$ death or $\mathrm{HF}$ hospitalization [hazard ratio (HR) in the LCZ696 group, 0.80; 95\% confidence interval (CI), 0.73-0.87; $p<0.001$ ) (Fig. 1) [2], a 20\% reduction in $\mathrm{CV}$ death (HR 0.80; 95\% CI $0.71-0.89 ; p<0.001)$ and a $16 \%$ reduction in the pre-specified key single end point of allcause mortality [HR 0.84 (95\% CI 0.76-0.93); $p<0.001]$. LCZ696 also reduced the risk of HF hospitalization by $21 \%(p<0.001)$ and decreased the symptoms and physical limitations of HF $(p=0.001)$. It is worth noting that the dose of enalapril used may have been suboptimal (maximum $10 \mathrm{mg}$ twice daily vs. the maximum valsartan dose of $320 \mathrm{mg}$ daily). The incidence of numerically greater, but non-significant excess of angioedema with LCZ696 may have been underestimated by use of a careful run-in period to exclude intolerant patients. Nevertheless, assuming LCZ696 receives a license, it is likely to be quickly included in clinical HF guidelines given its highly significant reduction in CV mortality and all-cause mortality.

The role of iron supplementation for patients with HF and anemia has been controversial. The Ferric Carboxymaltose evaluation on perFormance in patients with IRon deficiency in coMbination with chronic Heart Failure (CONFIRM HF) study (ClinicalTrials.gov \#NCT01453608) provided an important addition to the literature [3]. In this study, 304 HF patients (defined as LV ejection fraction $<45 \%$, NYHA II-III), with increased brain natriuretic peptide and serum ferritin $<100 \mathrm{ng} / \mathrm{mL}$ (or $100-300 \mathrm{ng} / \mathrm{mL}$ if transferrin saturation was $<20 \%$ ) were randomized to intravenous

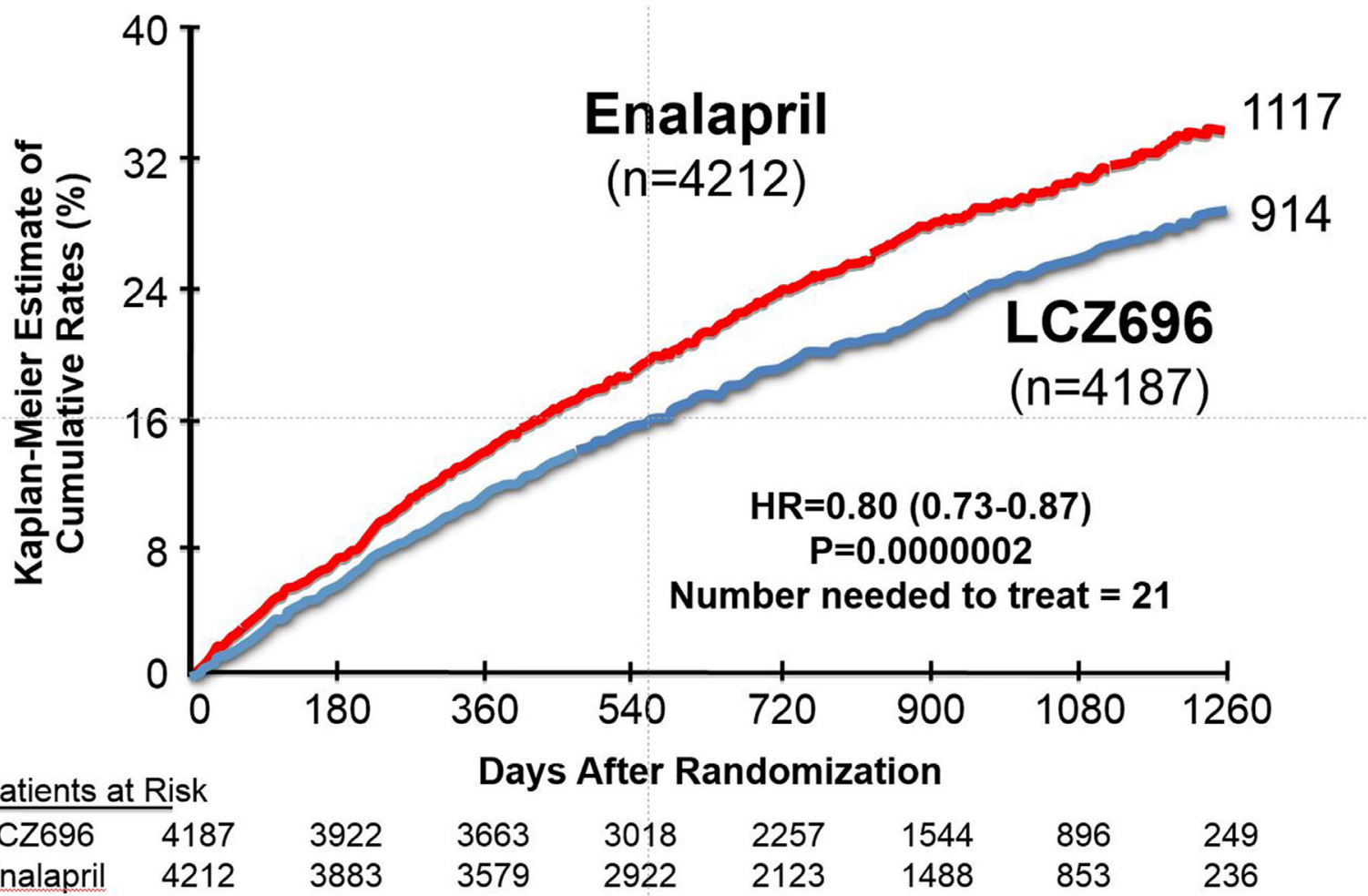

Fig. 1 The primary end point of cardiovascular death or HF hospitalization taken with permission from PARADIGM-HF. Reproduced with permission from [2]. HR hazard ratio 
ferric carboxymaltose or placebo given at time points of baseline, $6,12,24$, and 36 weeks. The trial's primary end point, the 6-minute walk test duration at 24 weeks, was significantly improved in the intravenous iron group (33 $\mathrm{m}$ greater distance; $p=0.002$ ), as were the secondary outcomes of NYHA class, fatigue score, quality of life scores, and self-reported patient global health assessment $(p<0.05$ for all). Treatment with intravenous iron was also associated with a significant reduction in the risk of $\mathrm{HF}$ hospitalizations [HR 0.39 (95\% CI 0.19-0.82); $p=0.009]$. The study was not powered to detect difference in mortality. In keeping with previous studies, CONFIRM HF supports iron treatment in HF patients with low ferritin.

A recurrent concern of HF patients is the presence of hyperkalemia. Reasons for this include concomitant renal failure and the use of drugs such as renin-angiotensin-aldosterone inhibiting agents and aldosterone antagonists. A potassium-absorbing compound, patiromer, was used in a two-phase trial, with hyperkalemic patients randomized to either placebo or this new drug with the dose depending on their initial serum potassium [4]. In the initial single-blind phase of the study, 102 patients with HF and 141 without received patiromer at two different dosage levels. Compared with placebo, there was a sustained decrease in serum potassium, both in patients with $(p<0.001)$ and without $(p<0.001)$ HF. The second phase of the study looked at patients who had received patiromer and who were taking a renin-angiotensinaldosterone inhibiting agent. In this phase, 107 patients whose initial potassium was $>5.5$ to $<6.5 \mathrm{mEq} / \mathrm{L}$ and subsequently decreased to 3.8 to $<5.5 \mathrm{mEq} / \mathrm{L}$ were randomized to continue patiromer $(n=55)$ or to switch to placebo $(n=52)$ and were followed for another 8 weeks. Switching patiromer to placebo led to a significant increase in potassium compared with maintenance, both in patients with $(n=49)$ and without $(n=58) \quad$ HF, with potassium levels $\geq 5.5 \mathrm{mmol} / \mathrm{l}$ occurring in $60 \%$ of the patients in the placebo group as compared to only $15 \%$ in the patiromer group $(p<0.001)$. Given these encouraging findings, further study with patiromer is planned including evaluation of the impact on overall clinical outcomes.

After an anterior wall myocardial infarction (MI), loss of myocardium results in an abrupt increase in loading conditions that induces remodeling of the LV [5]. Remodeling can lead to an increased LV volume, myocardial stress, and subsequent HF. To date, management options are limited for such patients. At the ESC Heart Failure Congress 2014 results of a pooled analysis of the first 111 patients treated with the Parachute device were presented [6]. The Parachute device is a percutaneous intervention that fits like an upside-down umbrella within the left ventricle apex aimed at improving ventricular function (Fig. 2) [7]. All patients presented had post-MI HF with anteroapical wall motion abnormalities. The results showed that the implantation of the device was successful in $95.5 \%$, and at 1 year there were a number of significant clinical and echocardiographic improvements. These included reductions in systolic and diastolic volumes, a moderate but significant rise in LV ejection fraction, and an improvement in 6-minute walk test distance and NYHA symptoms. Given these encouraging results, formal clinical outcome trials are now being planned.

\section{Percutaneous Coronary Intervention}

The optimum management of non-culprit lesions following primary percutaneous coronary intervention (PCI) in ST-elevation MI 


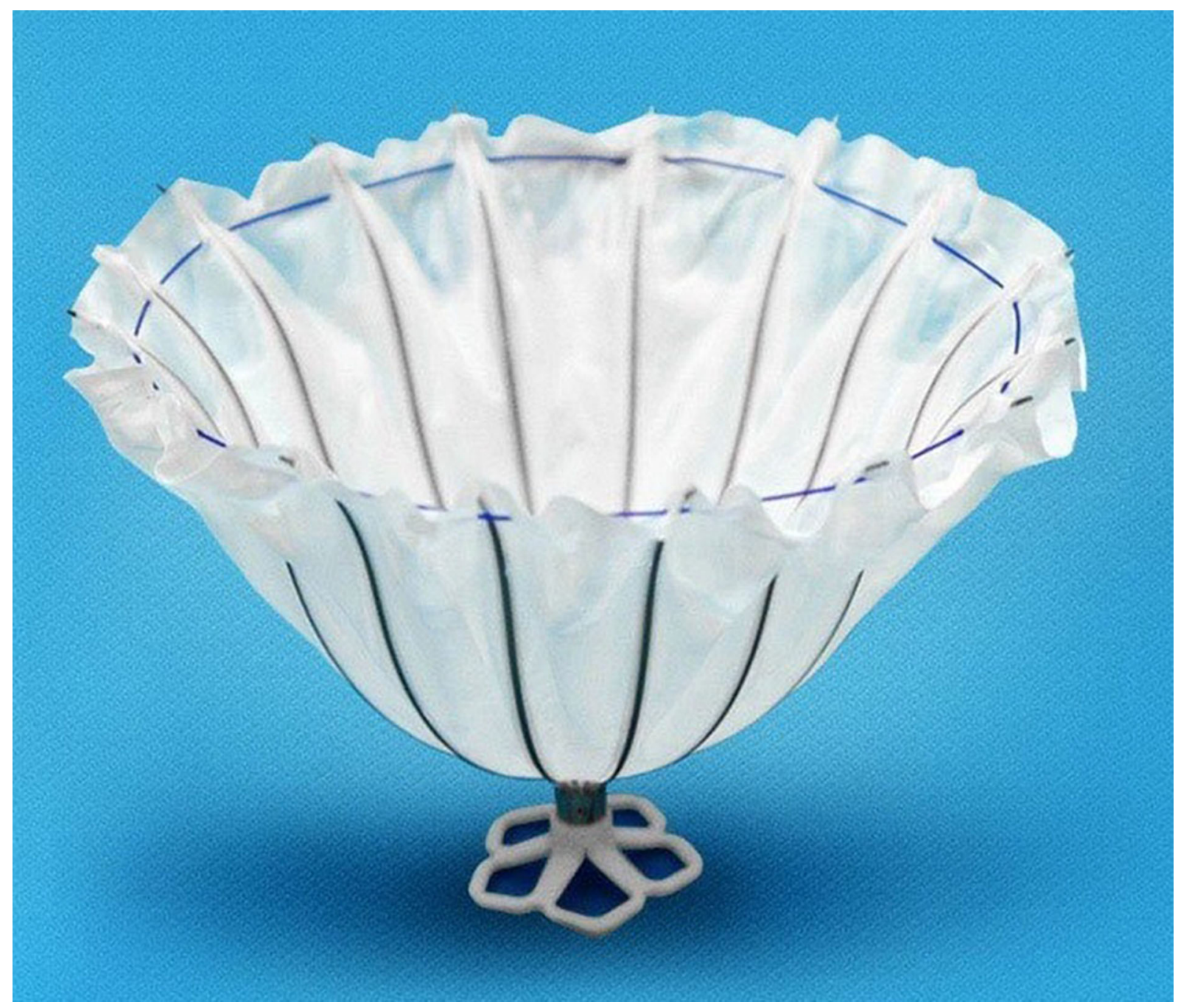

Fig. 2 The Parachute left ventricular device. Reproduced with permission from [7]

(STEMI) patients continues to be a focus of investigation. The previous Preventive Angioplasty in Acute Myocardial Infarction (PRAMI) trial (Current Controlled Trials \#ISRCTN73028481) [8]. reported that a strategy of non-culprit PCI during the same sitting as primary PCI, compared with no further intervention, reduced the composite end point of cardiac death, nonfatal MI, or refractory angina. However, concerns regarding PRAMI included possible overestimation of treatment effect due to early stopping of a small unblinded study. The Complete Versus Lesion-Only Primary PCI Trial (CVLPRIT) [9], which randomized patients to revascularization of all stenoses $>70 \%$ during index admission not necessarily at time of primary PCI $(n=150)$ vs. culprit-only PCI $(n=146), \quad$ also demonstrated significant reduction in the primary composite end point of total mortality, MI, HF, or ischemia-driven revascularization at 12 months $(10 \%$ vs. $21.2 \% ; p=0.009)$. Further analysis of PRAMI was presented at the SCAI 2014 meeting and 
reported that the severity of stenosis in nonculprit arteries was associated with future risk of subsequent clinical events [10]. Patients with an operator-determined $95-99 \%$ stenosis had a $47 \%$ incidence of major adverse cardiac events (MACE), whereas patients with 75-94\% stenosis and $50-74 \%$ stenosis had a $23 \%$ and $14 \%$ incidence of MACE, respectively. Although CVLPRIT was relatively small and there were no significant differences in mortality or HF (MACE difference being driven by soft end point of revascularization), along with PRAMI, it supports an interventional rather than conservative approach to non-culprit lesions. This is in contrast to a meta-analysis of 34,279 patients published earlier in 2014, which suggested that there was no significant difference in hospital mortality with multivessel PCI vs. culprit-only PCI [11]. Further prospective data are still required, which may be provided by the Complete vs. Culprit-only Revascularization to Treat Multivessel Disease After Primary PCI for STEMI (COMPLETE) study (ClinicalTrials.gov \#NCT01740479) which is a large ongoing trial comparing staged non-culprit PCI with conservative treatment [12].

The Thrombus Aspiration in ST-Elevation Myocardial Infarction in Scandinavia (TASTE) trial (ClinicalTrials.gov \#NCT01093404) presented its primary outcome (30 day mortality rates) at the 2013 ESC conference [13], and full 1-year results were presented at ESC 2014 [14]. This large, randomized, registry trial allocated 7244 patients to manual thrombus aspiration followed by PCI versus PCI only. There was no significant difference between the two groups in all-cause mortality at 1 year $(5.3 \%$ vs. 5.6\%; HR 0.94; 95\% CI, $0.78-1.15 ; p=0.57)$, rehospitalization for $\mathrm{MI}$ at 1 year $(2.7 \%$ vs. $2.7 \%$; HR $0.97 ; 95 \%$ CI
$0.73-1.28 ; p=0.81)$, stent thrombosis at 1 year (0.7\% vs. $0.9 \%$; HR $0.84 ; 95 \%$ CI $0.50-1.40$; $p=0.51)$, or the composite of death/MI/stent thrombosis, (8.0\% vs. $8.55 \%$; HR 0.94; $95 \% \mathrm{CI}$ $0.80-1.11 ; p=0.48)$. In contrast to previous data from the smaller TAPAS trial (Current Controlled Trials \#ISRCTN16716833) [15], TASTE did not support a role for routine thrombus aspiration before primary PCI.

The BIOSCIENCE trial (ClinicalTrials.gov \#NCT01443104) randomized 2119 patients undergoing PCI for chronic stable coronary artery disease or acute coronary syndromes to the ultrathin-strut $(60 \mu \mathrm{m})$ cobalt-chromium Orsiro stent (Biotronik, Berlin, Germany) releasing sirolimus from a biodegradable poly L-lactide polymer or to the thin-strut durable polymer everolimus-eluting stent Xience Prime (Abbott, Illinois, USA) [16]. The Orsiro stent met the definition of non-inferiority vs. Xience Prime for the primary composite end point of cardiac death/target vessel MI/clinically indicated target lesion revascularization at 12 months. $(6.5 \%$ vs. $6.6 \%$; $p$ for noninferiority $<0.0004)$. There was no difference in rates of definite stent thrombosis ( $0.9 \%$ vs. $0.4 \%$; $p=0.16)$, although longer-term follow-up beyond discontinuation of dual antiplatelet therapy is of more interest when evaluating possible benefits of a biodegradable polymer. Of note, in a pre-specified subgroup of patients with STEMI, the Orsiro stent was associated with a lower incidence of the primary end point [7 (3.3\%) vs. 17 (8.7\%), RR 0.38, 95\% CI 0.16-0.91; $p=0.024 ; \quad p$ for interaction $=0.014]$. This finding is consistent with previous biodegradable vs. durable polymer stents such as the Limus Eluted From A Durable Versus ERodable Stent Coating (LEADERS) trial (ClinicalTrials.gov \#NCT00389220) and warrants further study [17]. 


\section{Hypertension}

The role of renal denervation for resistant hypertension remains unclear. Several small open-label studies with different devices had reported treatment benefit for renal denervation, but the Renal Denervation in Patients With Uncontrolled Hypertension (SYMPLICITY HTN-3) trial (ClinicalTrials.gov \#NCT01418261) [18] which randomized 535 patients (2:1) to the first-generation Symplicity catheter (Medtronic, Santa Rosa, California, USA) versus a sham procedure failed to show a significant between-group difference in 6-month office blood pressure (BP) (14.1 vs. $11.7 \mathrm{mmHg} \quad$ reduction; between-group difference $2.39 \mathrm{mmHg}$; $95 \% \mathrm{CI}-6.89$ to 2.12 ; $p=0.26$; superiority margin $5 \mathrm{mmHg}$ ) or $24 \mathrm{~h}$ ambulatory BP (between group difference $-1.96 \mathrm{mmHg}$; $95 \% \mathrm{CI}-4.97$ to $1.06 ; p=0.98$, superiority margin $2 \mathrm{mmHg}$ ). A couple of reasons have been proposed for this lower than expected efficacy: firstly, heterogeneity in different patient subgroups; secondly possible suboptimal denervation due to device/operator factors as evident by lower BP reduction than previous studies and only $60 \%$ of patients in this study had only one or no ablation notches (arterial wall edema marks post-ablation) suggesting inadequate surface contact.

Patient selection also appears important. Six-month data from ongoing Global SYMPLICITY registry (ClinicalTrials.gov \#NCT01534299) of $>5000$ patients from $>200$ centers reported a $21.4 \mathrm{mmHg}$ reduction in patients with baseline systolic $\mathrm{BP} \geq 160 \mathrm{~mm} \mathrm{Hg}$, but only a $4.6 \mathrm{mmHg}$ reduction in those with baseline systolic BP 140-159 mmHg [19]. Greater numbers of effective ablation points correlated with benefit. Including treatment of accessory renal arteries also predicted better outcome. Further randomized studies with newer devices and studies to identify biomarkers predictive of procedural success are ongoing.

The Prevention Education Program (PEP) Family Heart Study was an important study in over 22,000 adolescents and children aged 3-18 years old [20]. Levels of BP were recorded and compared to the markers of obesity such as body mass index, waist circumference, waist-to-height ratio, percentage of body fat, and skinfold thickness. Pre-hypertension was defined as a BP between the 90th and 95th percentile of the BP curve for children and adolescents, while hypertension was a BP reading over the 95th percentile. The prevalence of hypertension was $5.4 \%$ in normal weight, $9.8 \%$ in overweight and $21.5 \%$ in obese subjects. Unsurprisingly, the presence of obesity strongly correlated with hypertension-obese boys having an odds ratio (OR) of 5.9 and obese girls 4.3. Compared with normal weight subjects, the risk of having pre-hypertension was significantly raised in overweight males and females (OR 1.6 and OR 1.8, respectively) and obese males and females (OR 2.4 and OR 3.3 , respectively). This study highlights the dangers of childhood obesity.

\section{Antiplatelet Therapy}

The optimum duration of dual antiplatelet therapy (DAPT) following coronary stenting or acute coronary syndrome has been assessed in several large studies during 2014. The DAPT study (ClinicalTrials.gov \#NCT00977938) presented at AHA 2014 randomized 9961 patients who had received a drug-eluting stent (DES) and 12 months of DAPT to aspirin only for a further 18 months (30 in total) of DAPT [21]. Those assigned to 30 months of DAPT 
showed significant reductions in co-primary end points of stent thrombosis [0.4\% vs. $1.4 \%$; HR 0.29 (95\% CI 0.17-0.48); $p<0.001$ ] and the composite of death/MI/stroke [4.3\% vs. 5.9\%; HR 0.71 (95\% CI 0.59-0.85); $p<0.001]$ and the secondary end point of MI $(2.1 \%$ vs. $4.1 \%$; HR $0.47 ; p<0.001)$. The risk of ischemic events increased markedly in the 3-month period after discontinuing thienopyridine treatment, regardless of when that occurred. However, 30 months therapy was associated with significantly greater moderate or severe bleeding (2.5\% vs. $1.6 \% ; p=0.001)$ and higher all-cause death [2.0\% vs. 1.5\%; HR 1.36 (95\% CI 1.00 to 1.85$) ; p=0.05]$ mainly due to non-CV deaths, including cancer deaths $(0.62 \%$ vs. $0.28 \% ; p=0.02$ ). The magnitude of benefit appeared highest in those receiving paclitaxeleluting stents $(27 \%$ of patients) in whom ongoing therapy may be appropriate, whereas shorter duration may be appropriate in newgeneration stents particularly if at increased bleeding risk.

The Second Generation Drug-Eluting Stent Implantation Followed by Six- Versus TwelveMonth Dual Antiplatelet Therapy (SECURITY) trial (ClinicalTrials.gov \#NCT00944333) randomized 1399 low-risk patients with stable or unstable angina or documented silent ischemia, undergoing PCI revascularization, with a second-generation DES to either 6 or 12 months of DAPT [22]. There was no significant difference between the two groups in the incidence of the primary composite end point (cardiac death, MI, stroke, definite or probable stent thrombosis or Bleeding Academic Research Consortium (BARC) type 3 or 5 bleeding at 12 months) occurring in $4.5 \%$ vs. $3.7 \%$ of patients, respectively; $p=0.469$ ). There was also no difference in the incidence of definite or probable stent thrombosis at
12 months ( $0.3 \%$ vs. $0.4 \% ; 95 \%$ CI -0.7 to $0.4 ; p=0.694)$. Interestingly there was no significant excess in bleeding with longer duration DAPT. The efficacy and safety data were confounded by $33 \%$ of patients in the 6-month group actually continuing therapy for 12 months.

However, these and previous data have helped to guide new ESC guidelines which recommend 6 months of DAPT following elective PCI with second-generation DES and consideration of only 3 months DAPT for patients at an increased risk of bleeding [23]. The Safety and Efficacy of 6 Months Dual Antiplatelet Therapy After Drug Eluting Stenting (ISAR-SAFE) study (ClinicalTrials.gov \#NCT00661206) also assessed the length of DAPT, suggesting that 6 months may be noninferior [24]. It compared patients with DES PCI who received 6 months of DAPT with aspirin and clopidogrel, followed by 1:1 randomization to an additional 6 months of either DAPT or aspirin alone. This trial was terminated prematurely due to a lower than anticipated event rate. A total of 4005 patients were randomized, 2007 to 12 months of DAPT and 1997 to 6 months of therapy. Baseline characteristics were similar between the two cohorts. The primary end point was MACE, which was non-significantly different between the 6- and 12-month DAPT arms. The composite of death, MI, stroke, and stent thrombosis was also similar $(1.3 \%$ vs. $1.5 \%$; $p=0.59)$ as were individual end points of mortality $(0.4 \%$ vs. $0.6 \% ; p=0.37)$, MI $(0.7 \%$ vs. $0.7 \% ; p=0.85)$, stent thrombosis $(0.3 \%$ vs. $0.2 \% ; p=0.74)$, and stroke $(0.4 \%$ vs. $0.3 \%$; $p=0.57)$. Thrombolysis in myocardial infarction (TIMI) major or minor bleeding was numerically lower with 6 months of DAPT $(0.3 \%$ vs. $0.7 \% ; p=0.12)$ and major bleeding 
(BARC $\geq 2)$ was significantly reduced ( $1 \%$ vs. $2 \% ; p=0.01)$. Overall, ISAR-SAFE suggested that 6 months of DAPT may be non-inferior to 12 months of DAPT with a trend toward lower bleeding. However, these results must be viewed with caution, as the study was halted prematurely due to a significantly lower event rate than anticipated (actual 1.6\% vs. anticipated 10\%).

The new P2Y12 antiplatelets ticagrelor and prasugrel achieve faster onset of action and approximately double the inhibition of platelet aggregation. Such properties might be expected to be of greatest benefit in acute coronary syndromes undergoing early intervention. In the study called A 30-day Study to Evaluate Efficacy and Safety of Prehospital vs. In-hospital Initiation of Ticagrelor Therapy in STEMI Patients Planned for Percutaneous Coronary Intervention (ATLANTIC) trial (ClinicalTrials.gov \#NCT01347580) [25], 1862 patients presenting in the community with STEMI and planned for primary PCI were randomized to immediate pre-hospital ticagrelor administration versus delayed ticagrelor administration in the cardiac catheterization laboratory. Unexpectedly, there was no difference in the co-primary end points of $>70 \%$ ST resolution or TIMI-3 flow between the two groups. However, platelet function analysis suggested that the intended early difference in platelet inhibition at the onset of primary PCI had not been achieved partly due to very rapid transfer of patients (only 31 min time difference) and a probable delayed absorption of ticagrelor in such acutely unwell patients, particularly if morphine had been given. A suggestion of more effective platelet inhibition with pre-hospital ticagrelor was noted $1 \mathrm{~h}$ post-PCI $(p=\mathrm{ns})$, but no difference by $6 \mathrm{~h}$ post-PCI. The pre-specified secondary end point of definite stent thrombosis was less in the pre-hospital group ( $0 \%$ vs. $0.8 \%$ in first $24 \mathrm{~h} ; p=0.008$ and $0.2 \%$ vs. $1.2 \%$ at 30 days; $p=0.02$ ). Rates of major bleeding events did not differ between the two groups. In summary, although there was no safety concern with pre-hospital use of ticagrelor, since the primary end point was not reduced, stent thrombosis observations cannot be regarded as definitive and at present, prehospital or in-hospital administration remains reasonable.

The Treatment With Adenosine Diphosphate (ADP) Receptor Inhibitors: Longitudinal Assessment of Treatment Patterns and Events After Acute Coronary Syndrome (TRANSLATEACS) observational study (ClinicalTrials.gov \#NCT01088503) enrolled 11,969 ACS patients undergoing PCI [26] who received either clopidogrel $(n=8846)$ or prasugrel $(n=3123)$. Unadjusted MACE rates (a composite of death, MI, stroke, or unplanned revascularization) were higher with clopidogrel than prasugrel $(17.3 \%$ vs. $13.5 \% ; \quad p<0.0001)$. However, patients receiving prasugrel were more likely to be younger, male, or presenting with STEMI and less likely to have had a history of prior MI, coronary artery bypass graft (CABG), or stroke/transient ischemic attack. After adjustment for baseline population differences, there was no longer any difference in overall MACE, although clopidogrel was associated with a higher risk of stent thrombosis but a lower risk of bleeding in keeping with previous randomized data.

\section{Anticoagulation}

The Explore the Efficacy and Safety of OnceDaily Oral Rivaroxaban for the Prevention of 
Cardiovascular Events in Patients With Nonvalvular Atrial Fibrillation Scheduled for Cardioversion (X-VeRT) trial (ClinicalTrials.gov \#NCT01674647) randomized 1504 patients with atrial fibrillation (AF) of presumed duration $>48 \mathrm{~h}$ and undergoing elective cardioversion [27] to the new oral anticoagulant rivaroxaban (20 mg once daily, $15 \mathrm{mg}$ if creatinine clearance $30-49 \mathrm{~mL} / \mathrm{min}$ ) or dose-adjusted warfarin in a 2:1 ratio. Additionally, investigators could randomize patients to either an early (target period of 1-5 days after randomization) or delayed (3-8 weeks) cardioversion strategy. The primary outcome (stroke, transient ischemic attack, peripheral embolism, MI, or CV death) occurred in 5 (two strokes) of 978 patients $(0.51 \%)$ receiving rivaroxaban (4 with early cardioversion and 1 with delayed cardioversion), compared with 5 (two strokes) of 492 patients $(1.02 \%)$ receiving warfarin (3 with early cardioversion and 2 with delayed cardioversion). Although the study was only exploratory and not powered to provide statistically rigorous results (which would have required $>10 \times$ the numbers), the findings are reassuring and in keeping with previous post hoc data from new oral anticoagulant trials.

\section{Transcatheter Aortic Valve Implantation}

Transcatheter aortic valve implantation (TAVI) has become standard cardiology practice in patients with severe aortic stenosis deemed not suitable for conventional surgery. Although initially greeted with skepticism, the importance of the procedure was recently highlighted by long-term follow-up of the Placement of Aortic Transcatheter Valves (PARTNER) study (ClinicalTrials.gov \#NCT00530894) [28] which reported a $47 \%$ reduction in 3-year mortality rate for TAVI compared with standard medical therapy $(54.1 \%$ vs. $80.9 \%$; HR 0.53 ; $95 \%$ CI $0.41-0.68 ; p<0.001)$.

Who should undergo TAVI has been a topic of debate addressed by a number of large trials. The multicenter randomized CoreValve HighRisk Study (ClinicalTrials.gov \#NCT01645202) randomized 795 high-risk severe aortic stenosis patients from 45 centers to TAVI or conventional surgery [29]. TAVI was performed using the Medtronic CoreValve group (323 via the iliofemoral route and 67 via non-iliofemoral access). The primary end point, 1 year all-cause mortality, was significantly lower with TAVI $(14.2 \%$ vs. $19.1 \% ; p=0.04)$. TAVI was non-inferior with respect to functional status and quality of life. As expected, the TAVI group had more major vascular complications, paravalvular leaks, and permanent pacemaker implantation, while the surgical group had higher rates of bleeding, acute kidney injury, and atrial fibrillation. The rate of stroke was not statistically different.

The Observational Study of Appropriateness, Efficacy and Effectiveness of AVR-TAVR Procedures for the Treatment of Severe Symptomatic Aortic Stenosis (OBSERVANT) observational cohort registry [30] enrolled intermediate risk patients with severe aortic stenosis and severe LV systolic dysfunction, eligible for TAVI $(n=650)$ or surgery $(n=650)$. Patients were matched for age, gender, baseline co-morbidities, previous interventions, priority at hospital admission, frailty score, and NYHA class. Exclusion criteria included requirement for concurrent revascularization, use of transapical TAVI, porcelain aorta, or hostile thorax. Despite the lower EuroScore surgical risk than in previous PARTNER [28] and CoreValve High-Risk studies [29], the 1 year OBSERVANT mortality rates presented at TCT [31] also showed no significant difference between TAVI and surgical groups (13.3\% vs. 
13.1\%; $\quad p=0.936), \quad$ MACE, $\quad$ cardiac rehospitalization, or HF admissions. The conclusions of the authors in this study suggest that in patients with severe LV systolic dysfunction, TAVI (at least via the transfemoral route) appears a reasonable alternative to conventional surgery. However, it is important to be cautious with regard to this, as the data presented are not randomized and not in keeping with the conventional view that aortic stenosis is best treated with surgical aortic valve replacement. The forthcoming Placement of AoRTic TraNscathetER Valves (PARTNER II) (ClinicalTrials.gov \#NCT01314313) and Safety and Efficacy Study of the Medtronic CoreValve ${ }^{\circledR}$ System in the Treatment of Severe, Symptomatic Aortic Stenosis in Intermediate Risk Subjects Who Need Aortic Valve Replacement (SURTAVI) (ClinicalTrials.gov \#NCT01586910) studies will help further evaluate TAVI in low- and intermediate-risk patients [32, 33].

\section{Stable Coronary Artery Disease}

Previous subgroup analysis suggested that the sino-atrial node-blocking agent ivabradine may improve outcomes in patients with stable coronary artery disease and a heart rate of 70 beats per minute or more, especially among those with limiting angina [34]. Study Assessing the Morbidity-Mortality Benefits of the $I_{f}$ Inhibitor Ivabradine in Patients with Coronary Artery Disease (SIGNIFY) (EudraCT \#2009011360-10) [35] randomized 19,102 patients with stable coronary artery disease without clinical HF, heart rate $\geq 70$ beats per minute to ivabradine or placebo. Over the 28.7 months follow-up, there was no difference in the primary end point of $\mathrm{CV}$ death or nonfatal MI between the cohorts $(6.8 \%$ and $6.4 \%$, respectively; HR 1.08; 95\% CI $0.96-1.20$; $p=0.20$ ). Unexpectedly, ivabradine was associated with an increase in the primary end point among patients with limiting angina (12,049 patients had Canadian Cardiovascular Society class $\geq$ II angina), but not among those without limiting angina $(p$ interaction $=0.02$ ). Ivabradine, which was at doses up to $10 \mathrm{mg}$ twice a day (higher than currently licensed), was associated with a marked increase in bradycardia ( $18.0 \%$ vs. $2.3 \%$; $p<0.001)$. While heart rate lowering remains of symptomatic value for stable angina, this important and wellconducted trial challenges the commonly held view regarding prognostic benefit. In particular, excessive heart rate lowering may be disadvantageous.

The value of invasive fractional flow reserve (FFR) assessment of moderate coronary stenoses is gaining increasingly wide clinical acceptance. The multicenter Portuguese Study on the Evaluation of FFR Guided Treatment of Coronary Disease (POST-IT) registry (ClinicalTrials.gov \#NCT01835808) [36] at EuroPCR 2014 described how, compared with standard quantitative coronary angiography alone, use of FFR changed physicians' choice of treatment in $44 \%$ of 918 patients from 19 centers. Specifically, while the overall number of PCI did not change significantly, the number of elective CABG referrals declined by almost 50\%.

In the Fractional Flow Reserve (FFR) Guided Percutaneous Coronary Intervention Plus Optimal Medical Treatment Versus Optimal Medical Therapy alone (FAME II) trial (ClinicalTrials.gov \#NCT01132495), stable patients $(n=1220)$ scheduled for elective PCI first underwent pressure wire assessment for FFR [37]. Those patients $(n=888)$ with at least one coronary lesion FFR $\leq 0.80$ were randomized to PCI plus medical therapy versus medical therapy alone. Those with FFR $>0.80$ were not randomized, but received medical therapy only 
and registry follow-up. Within the FFR $\leq 0.80$ group, PCI plus medical therapy versus medical therapy alone was associated with significant reduction in the primary end point of death, MI, or urgent revascularization ( $8.1 \%$ vs. $19.5 \%$; HR 0.39; 95\% CI 0.26-0.57; $p<0.001$ ) driven by a lower rate of urgent revascularization. Although there were no significant differences in the rates of death and MI overall, in a landmark analysis the rate of death or MI from 8 days to 2 years was lower in the PCI group than in the medical therapy group $(4.6 \%$ vs. $8.0 \% ; p=0.04)$. Of note, the use of PCI reduced event rates similar to that seen in the registry of patients with non-obstructive disease (FFR $>0.80$ ) in whom the primary end point was $9.0 \%$ at 2 years.

\section{Arrhythmias and Devices}

Cardiac resynchronization therapy (CRT) has been proven to improve HF symptoms and outcomes in patients with LV dysfunction and a broad QRS complex on electrocardiography [38, 39]. However, failure to implant an LV lead occurs in approximately $5-15 \%$ of cases $[40,41]$. Reasons for this include unsatisfactory pacing parameters and phrenic nerve stimulation (PNS). The presence of more electrodes on the LV lead may allow a change in pacing vector which may reduce PNS and unsatisfactory pacing capture thresholds. The multicenter More Options Available with a Quadripolar LV Lead Provide In-clinic Solutions to CRT Challenges (MORE CRT) trial (ClinicalTrials.gov \#NCT01510652) randomized 1068 patients undergoing CRT to receive quadripolar $(n=720)$ or bipolar $(n=348)$ leads [42]. Patients were evaluated for intraoperative events (including PNS, lead instability, high capture threshold leading to change in tributary vein of coronary sinus after target-site evaluation, use of more than one LV lead during the procedure, requirement for active lead fixation, or unsuccessful implantation) and postoperative events (related to the LV lead or abandonment of CRT). Use of quadripolar leads compared with bipolar leads was associated with a 40.8\% reduction in the primary composite end point of intra- and postoperative LV lead-related events (13.2\% vs. $22.3 \% ; p=0.0002)$. Although the study was not powered to evaluate subsequent clinical outcomes, a quadripolar lead strategy appears promising.

Use of pacemakers without leads may potentially reduce transvenous and subcutaneous complications (Fig. 3) [43]. The LEADLESS registry (ClinicalTrials.gov \#NCT02051972) enrolled 33 patients undergoing leadless pacemaker implantation with the primary end point being 90 days complication rate [44]. The implant success rate was $97 \%(n=32)$. One patient developed right ventricular perforation and cardiac tamponade during the implant procedure, and eventually died as a result of a stroke. The overall complication-free rate was $94 \%$. After 90 days, pacing performance was within the accepted range. No patient required a revision of the system (following the index procedure), and all implants demonstrated an adequate safety margin in nominal pacing amplitude and sensing threshold. This proof of concept study suggests that single-chamber leadless cardiac pacemaker implantation appears feasible. Larger studies are planned to further evaluate the efficacy and safety compared with conventional lead-based systems.

The importance of antithrombotic therapy in incidentally detected ambulatory atrial fibrillation (IA-AF) has been recently highlighted [45]. Martinez et al. compared the 3-year incidence of stroke, all-cause mortality, MI, and major bleeding in 5555 patients with IA-AF and in 24,705 age- and 


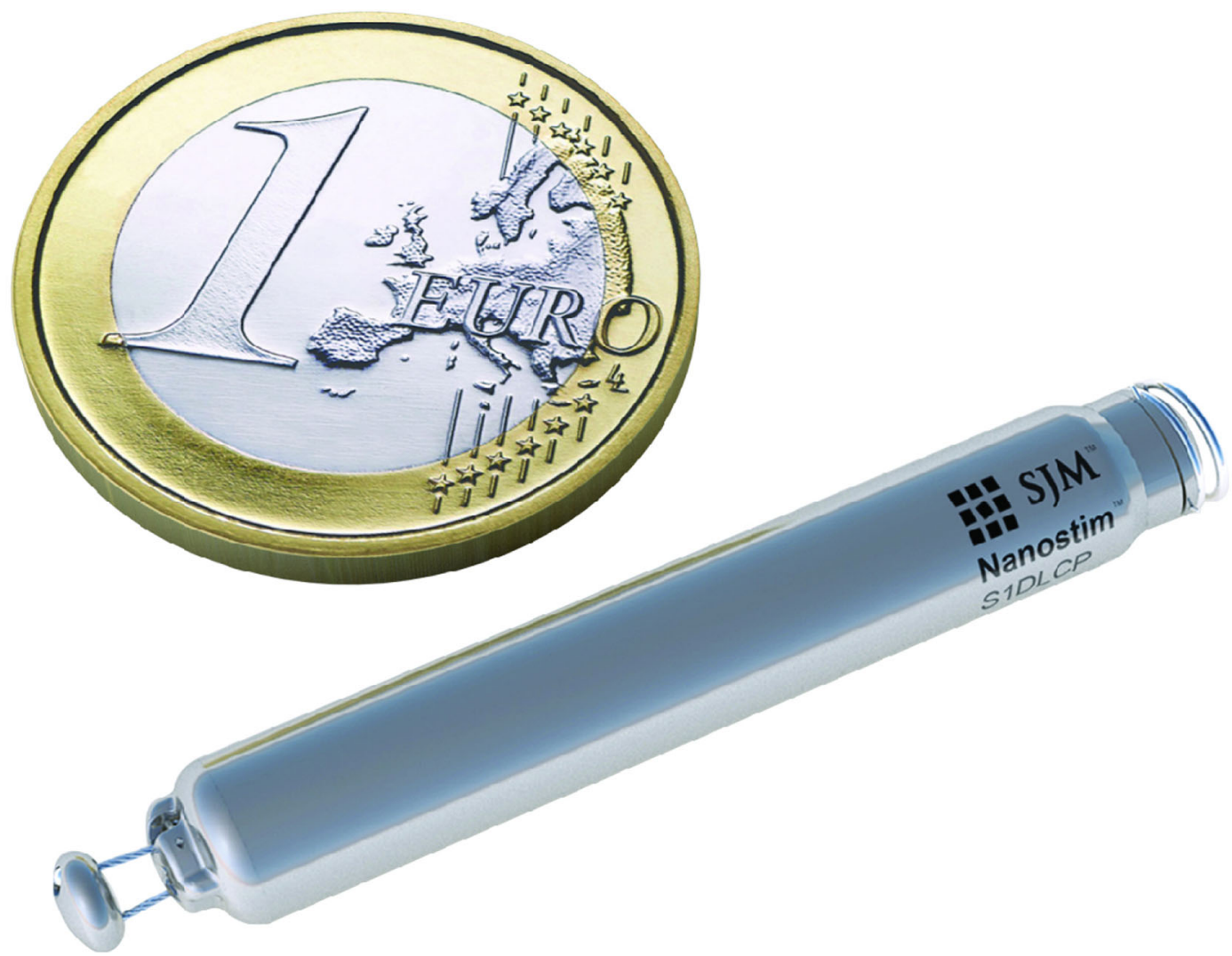

Fig. 3 Leadless pacemaker, with slide showing its actual size. Nanostim and St. Jude Medical are trademarks of St. Jude Medical, Inc. or its related companies. Reprinted with permission of [43]

gender-matched controls without AF. Patients with IA-AF had mean CHA2DS2VASc score $2.5 \pm 1.5$. Those with IA-AF had a significantly higher incidence of stroke (19.4 vs. $8.4 / 1000$ person-years; $p<0.001)$, mortality (40.1 vs. 20.9/1000 person-years; $p<0.001$ ), and MI (9.0 vs. $6.5 / 1000$ person-years; $p<0.001)$. In the year following IA-AF detection, the use of oral anticoagulant (received by 51.0\%) was associated with reduced stroke [adjusted HR 0.35 (0.17-0.71)] and death [adjusted HR 0.56 (0.36-0.85)]. Antiplatelet treatment was only associated with a non-significant reduction of stroke and death. Both treatments carried a small non-significant adjusted incidence of major bleeding. This study supports the association of asymptomatic AF with adverse outcomes, which may be reduced by oral anticoagulant but not antiplatelet treatment.

An interesting, yet small study presented at the ESC 2014 by Anguera et al. [46] involved quinidine administration in Brugada syndrome patients receiving regular appropriate implantable cardioverter defibrillator shocks. The cohort was small with only 23 patients, with a mean age $41 \pm 13$ years and 19 (82\%) being male. After a mean follow-up of $47 \pm 43$ months using quinidine, there was a significant reduction in the median number of shocks from five shocks per patient [interquartile range (IQR) 3, 5-7] to a median of zero (IQR 0-0). Side effects were seen in four $(17 \%)$ patients who were managed with 
reduction of the doses of quinidine. Thus in patients with Brugada syndrome and recurrent ventricular shocks, it may be reasonable to consider quinidine administration to try and reduce shock frequency.

\section{Atherosclerosis}

Until now, the clinical value of the non-statin lipid-lowering agent ezetimibe has been unclear. It typically reduces low-density lipoprotein cholesterol (LDL-C) by $15-20 \%$ and is usually well tolerated, but did not reduce progression of carotid intima media thickness in a previous study of patients with familial hyperlipidemia. Examining Outcomes in Subjects With Acute Coronary Syndrome: Vytorin (Ezetimibe/ Simvastatin) vs. Simvastatin (IMPROVE-IT; ClinicalTrials.gov \#NCT00202878) [47] randomized 18144 patients with ACS $\leq 10$ days and low LDL-C $(<2.6 \mathrm{mmol} / \mathrm{l}$ if prior lipidlowering therapy; otherwise $<3.2 \mathrm{mmol} / \mathrm{l}$ ) to simvastatin (40 or $80 \mathrm{mg}$ ) versus simvastatin plus ezetimibe $10 \mathrm{mg}$ aiming for LDL-C $<2.0 \mathrm{mmol} / \mathrm{l}$. IMPROVE-IT was designed to test two hypotheses: (1) if lowering LDL-C is better for CV outcomes even at very low baseline LDL-C and (2) if adding another non-statin LDLlowering drug to a statin reduces $\mathrm{CV}$ outcomes. Interim analysis guided the increase in study size (originally 10,000 patients) and follow-up was extended up to 7 years until at least 5250 subjects experienced a primary end-point first event (CV death, MI, re-hospitalization for unstable angina, coronary revascularization, or stroke). At baseline, the mean LDL-C level was $2.4 \mathrm{mmol} / \mathrm{l}$ in both treatment arms. At 1 year, simvastatin alone (27\% uptitrated to $80 \mathrm{mg}$ ) lowered LDL-C to a mean of $1.8 \mathrm{mmol} / \mathrm{l}$, whereas simvastatin (6\% uptitrated to $80 \mathrm{mg}$ ) plus ezetimibe lowered LDL-C to a mean of $1.4 \mathrm{mmol} / \mathrm{l}$. The well-treated population (already at low LDL-C, with both study arms receiving statin) and study prolongation (increasing drop outs/loss to follow-up and the impact of recurrent vascular events) suggested it might be difficult to meet the primary end point. Despite this, addition of ezetimibe to simvastatin reduced the primary end point by $6.4 \%$ (34.7\% vs. $32.7 \% ; p=0.016)$ (Fig. 4), driven by a significant reduction in MI (14.8\% vs. $13.1 \% ; p=0.002)$ and ischemic stroke (4.1\% vs. $3.4 \% ; p=0.008)$ (Fig. 5). Although absolute risk reduction was not marked, IMPROVE-IT is a key trial, being the first to demonstrate incremental clinical benefit when adding a non-statin agent (ezetimibe) to statin therapy.

The Study of Alirocumab (REGN727/ SAR236553) in Patients With Primary Hypercholesterolemia and Moderate, High, or Very High Cardiovascular (CV) Risk, Who Are Intolerant to Statins (ODYSSEY ALTERNATIVE; ClinicalTrials.gov \#NCT01709513) trail [48] evaluated the PCKS9 monoclonal antibody alirocumab patients with statin intolerance and LDL-C $>1.8 \mathrm{mmol} / \mathrm{l}$ if very high $\mathrm{CV}$ risk or $\mathrm{LDL}>2.6 \mathrm{mmol} / \mathrm{l}$ if moderate/high risk. Mean baseline LDL-C was $4.9 \mathrm{mmol} / \mathrm{l}$. Following a 4-week placebo run-in phase (during which 47 patients dropped out because of muscle-related symptoms), 314 patients were randomized to subcutaneous injection with alirocumab $75-150 \mathrm{mg}$ every 2 weeks $(n=126)$, ezetimibe $10 \mathrm{mg}(n=125)$ or atorvastatin $20 \mathrm{mg}(n=63)$. Alirocumab lowered LDL-C levels significantly more than ezetimibe by 24 weeks (intention to treat $-45.0 \%$ vs. $-14.6 \%$; $p<0.0001$ and ontreatment $-52.2 \%$ vs. $-17.1 \% ; p<0.0001)$. Alirocumab was better tolerated than atorvastatin and produced greater LDL reductions. Given the positive results of a nonstatin drug ezetimibe in IMPROVE-IT, it is thus 


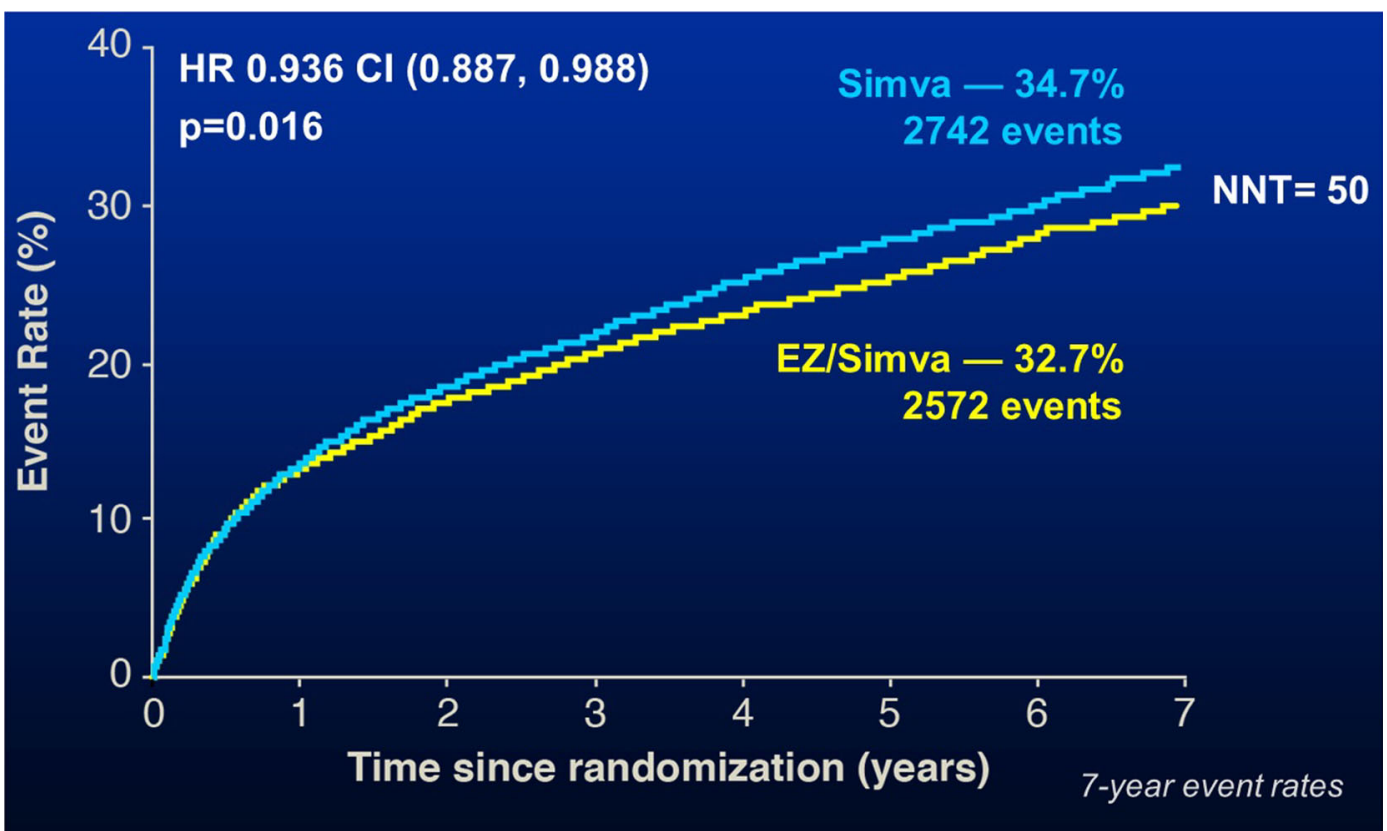

Fig. 4 IMPROVE-IT primary end point: cardiovascular death, MI, documented unstable angina requiring rehospitalization, coronary revascularization ( $\geq 30$ days), or stroke.
Reproduced with permission from [47]. CI confidence interval, $E Z$ ezetimibe, $H R$ hazard ratio, $N N T$ numbers needed to treat, Simva simvastatin

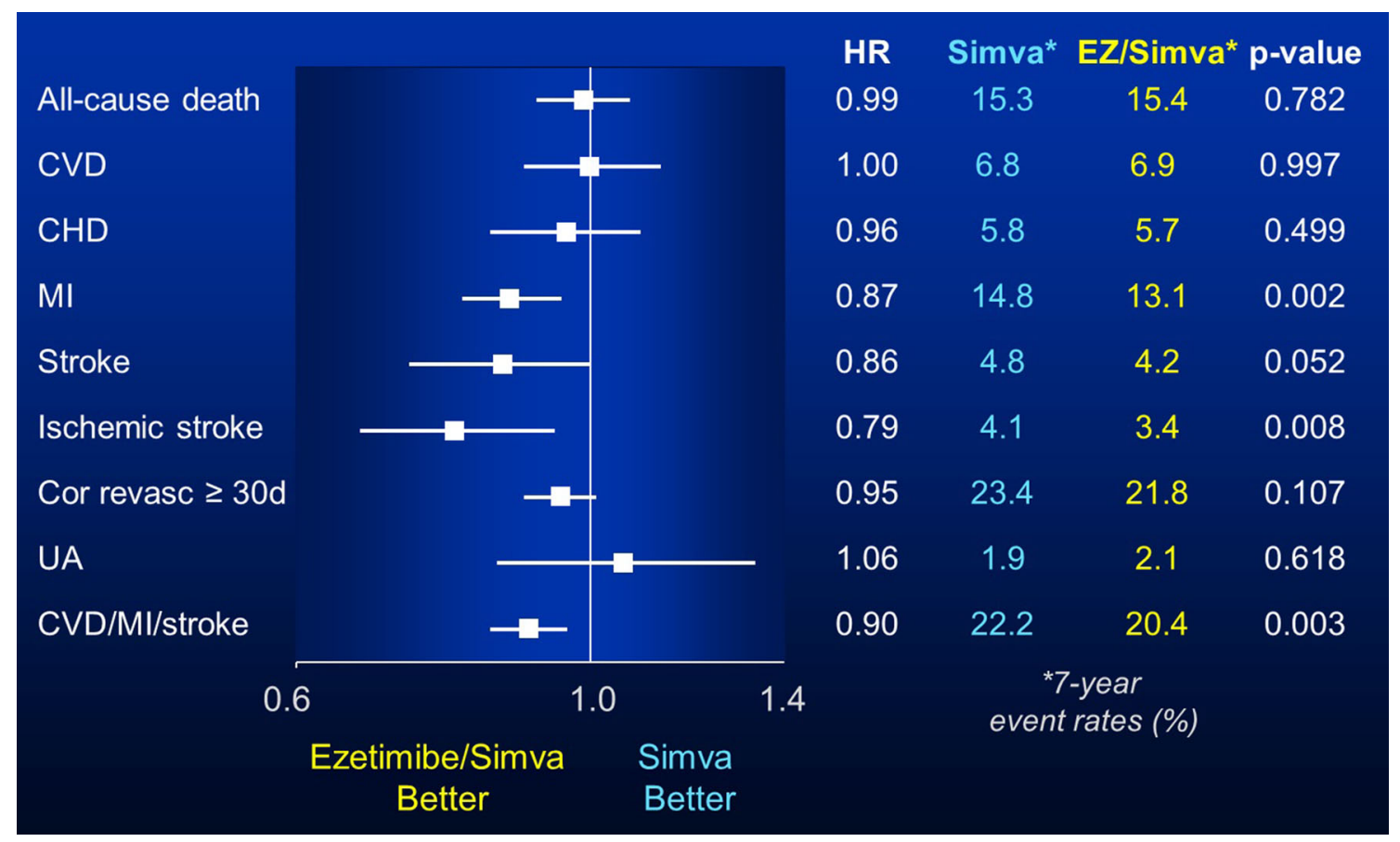

Fig. 5 IMPROVE-IT: individual cardiovascular end points and CVD/MI/stroke. Reproduced with permission from [47]. CHD coronary heart disease, Cor revasc coronary artery revascularization, $C V D$ cardiovascular disease, $E Z$ ezetimibe, $H R$ hazard ratio, $M I$ myocardial infarction, Simva simvastatin, $U A$ unstable angina 
hoped that PCSK9 inhibitors will also be associated with clinical benefit in ongoing $\mathrm{CV}$ outcome trials.

The value in cardiac screening in asymptomatic patients has long been controversial [49]. The Screening For Asymptomatic Obstructive Coronary Artery Disease Among High-Risk Diabetic Patients Using CT Angiography, Following Core 64 (FACTOR-64) trial (ClinicalTrials.gov \#NCT00488033) randomized 900 diabetic patients to either cardiac computerized tomography angiography (CCTA) screening $(n=452)$ or to standard national guidelinesbased optimal diabetes care $(n=448)$. Those in the CCTA arm could receive subsequent invasive coronary angiography, based on CCTA findings. After a mean follow-up of 4 years, the primary outcome (all-cause mortality, nonfatal MI, or unstable angina requiring hospitalization) showed a small, but non-significant reduction with CCTA screening [6.2\% (28 events) vs. 7.6\% (34 events); HR 0.80 (95\% CI 0.49-1.32); $p=0.38]$. At present, such data do not support routine CCTA screening in high-risk diabetic patients, although larger studies with greater power are being undertaken.

\section{CONCLUSION}

Over the past year, a number of important advances have been presented in major international cardiology meetings. Some findings are ready to influence clinical practice, while others require additional discussion and integration, or further work, before clinical adoption. This review has highlighted the most important trials, thus giving an overview of the most important breaking research from 2014.

\section{ACKNOWLEDGMENTS}

No funding or sponsorship was received for this study or publication of this article. All named authors meet the International Committee of Medical Journal Editors (ICMJE) criteria for authorship for this manuscript, take responsibility for the integrity of the work as a whole, and have given final approval for the version to be published.

Conflict of interest. Peter McKavanagh and Claire McCune declare they have no conflicts of interest. Ian Menown has received grants to institution, honoraria and/or conference sponsorship from: Boston Scientific, Sanofi Aventis, Biosensors, Meril Life, Orbus Neich, Menarini, Astra Zeneca, Bayer, Boehringer Ingelheim, Daichii Sankyo, Lilly, Bristol Myers Squibb, and Pfizer.

Compliance with ethics guidelines. This article is based on previously conducted studies and does not involve any new studies of human or animal subjects performed by any of the authors.

Open Access. This article is distributed under the terms of the Creative Commons Attribution Noncommercial License which permits any noncommercial use, distribution, and reproduction in any medium, provided the original author(s) and the source are credited.

\section{REFERENCES}

1. Fuster V, van der Zee S, Elmariah S, Bonow RO. Careers in cardiovascular research. Circulation. 2009;119:754-60.

2. McMurray JJ, Packer M, Desai AS, Gong J, Lefkowitz MP, Rizkala AR, PARADIGM-HF Investigators and 
Committees, et al. Angiotensin-neprilysin inhibition versus enalapril in heart failure. N Engl J Med. 2014;371(11):993-1004.

3. Ponikowski P, van Veldhuisen DJ, Comin-Colet J, Ertl G, Komajda M, Mareev V, et al. for the CONFIRM-HF Investigators. Beneficial effects of long-term intravenous iron therapy with ferric carboxymaltose in patients with symptomatic heart failure and iron deficiency. Eur Heart J. 2014. pii:ehu385 (Epub ahead of print).

4. Weir MR, Bakris GL, Bushinsky DA, Mayo MR, Garza D, Stasiv Y, Wittes J, Christ-Schmidt H, Berman L, Pitt B; the OPAL-HK Investigators. Patiromer in patients with kidney disease and hyperkalemia receiving RAAS inhibitors. N Engl J Med. 2014.

5. Sutton MG, Sharpe N. Left ventricular remodeling after myocardial infarction: pathophysiology and therapy. Circulation. 2000;101(25):2981-8.

6. Abraham W. Percutaneous ventricular restoration therapy using the parachute device in patients with ischemic dilated heart failure: pooled analysis of the first 100 patients treated for 1 year. In: Presented at European Society of Cardiology Heart Failure Association, May 19, Athens, Greece. 2014.

7. CardioKinetix, Inc. The Parachute left ventricular device. Reproduced with permission from www. cardiokinetix.com. Accessed 28 Feb 2015.

8. Wald DS, Morris JK, Wald NJ, Chase AJ, Edwards RJ, Hughes LO, et al. PRAMI Investigators. Randomized trial of preventive angioplasty in myocardial infarction. N Engl J Med. 2013;369(12):1115-23.

9. Gershlick AH. Complete versus Lesion only PRimary -PCI Trial (CvLPRIT): treat the infarct related artery only or all lesions. In: Presented at European Society of Cardiology, September 1, Barcelona, Spain. 2014.

10. Oldroyd, K. Preventive Angioplasty in Acute Myocardial Infarction. In: Presented at Society for Cardiovascular Angiography and Interventions (SCAI) 2014 Annual Scientific Sessions, May 30, Las Vegas, Nevada, USA. 2014.

11. Bainey KR, Mehta SR, Lai T, Welsh RC. Complete vs culprit-only revascularization for patients with multivessel disease undergoing primary percutaneous coronary intervention for STsegment elevation myocardial infarction: a systematic review and meta-analysis. Am Heart J. 2014;167(1):1-14.

12. Complete versus culprit-only revascularization strategies to treat multi-vessel disease after primary percutaneous coronary interventions for
ST-segment elevation myocardial infarction (COMPLETE) trial. http://www.controlled-trials. com/isrctn/pf/21662248. Accessed 28 Feb 2015.

13. Fröbert $\mathrm{O}$, Lagerqvist $\mathrm{B}$, Olivecrona GK, Omerovic E, Gudnason T, Maeng $M$, et al. Thrombus aspiration during ST-segment elevation myocardial infarction (TASTE Trial). N Engl J Med. 2013;369(17):1587-97.

14. Lagerqvist B, Fröbert O, Olivecrona GK, Gudnason T, Maeng M, Alström P, et al. Outcomes 1 year after thrombus aspiration for myocardial infarction. N Engl J Med. 2014;371(12):1111-20.

15. Svilaas T, van der Horst IC, Zijlstra F. Thrombus Aspiration during Percutaneous coronary intervention in Acute myocardial infarction Study (TAPAS)-study design. Am Heart J. 2006;151(3):597.e1-7.

16. Pilgrim $T$, Heg $D$, Roffi $M$, Tüller $D$, Muller $O$, Vuilliomenet A, et al. Ultrathin strut biodegradable polymer sirolimus-eluting stent versus durable polymer everolimus-eluting stent for percutaneous coronary revascularisation (BIOSCIENCE): a randomised, single-blind, non-inferiority trial. Lancet. 2014. pii:S0140-6736(14)61038-2 (epub ahead of print).

17. Serruys PW, Farooq V, Kalesan B, de Vries $T$, Buszman P, Linke A, Ischinger T, Klauss V, Eberli F, Wijns W, Morice MC, Di Mario C, Corti R, Antoni D, Sohn HY, Eerdmans P, Rademaker-Havinga T, van Es GA, Meier B, Jüni P, Windecker S. Improved safety and reduction in stent thrombosis associated with biodegradable polymer-based biolimus-eluting stents versus durable polymerbased sirolimus-eluting stents in patients with coronary artery disease: final 5-year report of the LEADERS (Limus Eluted From A Durable Versus ERodable Stent Coating) randomized, noninferiority trial. JACC Cardiovasc Interv. 2013;6(8):777-89.

18. Bakris GL, Townsend RR, Liu M, Cohen SA, D'Agostino R, Flack JM, et al. Impact of renal denervation on 24-hour ambulatory blood pressure: results from SYMPLICITY HTN-3. J Am Coll Cardiol. 2014;64(11):1071-8.

19. Böhm M, Mahfoud F, Ukena C, Hoppe UC, Narkiewicz K, Negoita M, Ruilope L, Schlaich MP, Schmieder RE, Whitbourn R, Williams B, Zeymer U, Zirlik A, Mancia G, on behalf of the GSR Investigators. First Report of the Global SYMPLICITY Registry on the effect of renal artery denervation in patients with uncontrolled hypertension. Hypertension. 2015;17(114):05010.

20. Schwandt P, Scholze JE, Bertsch T, Liepold E, Haas GM. Blood pressure percentiles in 22,051 German 
children and adolescents: the PEP Family Heart Study. Am J Hypertens. 2014. pii:hpu208 (epub ahead of print).

21. Mauri L, Kereiakes DJ, Yeh RW, et al. Twelve or 30 months of dual antiplatelet therapy after drugeluting stents. N Engl J Med. 2014;371:2155-66.

22. Colombo A, Chieffo A, Frasheri A, Garbo R, Masotti $\mathrm{M}$, Salvatella $\mathrm{N}$, et al. Second generation drugeluting stents implantation followed by six versus twelve-month-dual antiplatelet therapy-the Security Randomized Clinical Trial. J Am Coll Cardiol. 2014. Pii:S0735-1097(14)06155-5.

23. Windecker S, Kolh P, Alfonso F, Collet JP, Cremer J, Falk V, et al. 2014 ESC/EACTS Guidelines on myocardial revascularization: the Task Force on Myocardial Revascularization of the European Society of Cardiology (ESC) and the European Association for Cardio-Thoracic Surgery (EACTS) Developed with the special contribution of the European Association of Percutaneous Cardiovascular Interventions (EAPCI). Eur Heart J. 2014;35(37):2541-619.

24. Schulz-Schüpke S, Byrne RA, Ten Berg JM, Neumann FJ, Han Y, Adriaenssens T, Tölg R, Seyfarth M, Maeng M, Zrenner B, Jacobshagen C, Mudra H, von Hodenberg E, Wöhrle J, Angiolillo DJ, von Merzljak B, Rifatov N, Kufner S, Morath T, Feuchtenberger A, Ibrahim T, Janssen PW, Valina C, Li Y, Desmet W, Abdel-Wahab M, Tiroch K, Hengstenberg C, Bernlochner I, Fischer M, Schunkert H, Laugwitz KL, Schömig A, Mehilli J, Kastrati A; on behalf of the Intracoronary Stenting and Antithrombotic Regimen. Safety and Efficacy of 6 Months Dual Antiplatelet Therapy After DrugEluting Stenting (ISAR-SAFE) trial investigators. ISAR-SAFE: a randomized, double-blind, placebocontrolled trial of 6 versus 12 months of clopidogrel therapy after drug-eluting stenting. Eur Heart J. 2015. pii:ehu523 (epub ahead of print).

25. Montalescot $\mathrm{G}$, van 't Hof AW, Lapostolle F, Silvain J, Lassen JF, Bolognese L, et al; ATLANTIC Investigators. Prehospital ticagrelor in ST-segment elevation myocardial infarction. $\mathrm{N}$ Engl J Med. 2014;371(11):1016-27.

26. Bagai A, Peterson ED, Honeycutt E, Effron MB, Cohen DJ, Goodman SG, Anstrom KJ, Gupta A, Messenger JC, Wang TY. In-hospital switching between adenosine diphosphate receptor inhibitors in patients with acute myocardial infarction treated with percutaneous coronary intervention: insights into contemporary practice from the TRANSLATE-ACS study. Eur Heart J Acute Cardiovasc Care. 2014. pii:2048872614564082 (epub ahead of print).
27. Cappato R, Ezekowitz MD, Klein AL, Camm AJ, Ma CS, Le Heuzey JY, et al; on behalf of the $\mathrm{X}$-VeRT Investigators. Rivaroxaban vs. vitamin $\mathrm{K}$ antagonists for cardioversion in atrial fibrillation. Eur Heart J. 2014. pii:ehu367. (epub ahead of print).

28. Kapadia SR, Tuzcu EM, Makkar RR, Svensson LG, Agarwal S, Kodali S, et al. Long-term outcomes of inoperable patients with aortic stenosis randomly assigned to transcatheter aortic valve replacement or standard therapy. Circulation. 2014;130(17):1483-92.

29. Adams DH, Popma JJ, Reardon MJ, Yakubov SJ, Coselli JS, Deeb GM, U.S. CoreValve Clinical Investigators, et al. Transcatheter aortic-valve replacement with a self-expanding prosthesis. N Engl J Med. 2014;370(19):1790-8.

30. Capodanno D, Barbanti M, Tamburino C, D'Errigo P, Ranucci M, Santoro G, OBSERVANT Research Group, et al. A simple risk tool (the OBSERVANT score) for prediction of 30-day mortality after transcatheter aortic valve replacement. Am J Cardiol. 2014;113(11):1851-8.

31. Barbanti M. The OBSERVANT registry 1 year result. In: Presented at Transcatheter Cardiovascular Therapeutics, September 16, Washington, DC, USA. 2014.

32. The PARTNER II Trial: Placement of AoRTic TraNscathetER Valves. http://clinicaltrials.gov/ ct $2 /$ show/NCT01314313?term =partner+AND+ sapien\&rank=2. Accessed 28 Feb 2015.

33. Safety and Efficacy Study of the Medtronic CoreValve $^{\circledR}$ System in the Treatment of Severe, Symptomatic Aortic Stenosis in Intermediate Risk Subjects Who Need Aortic Valve Replacement (SURTAVI). http://clinicaltrials.gov/ct2/show/NCT 01586910 ?term $=$ SURTAVI\&rank=1. Accessed 28 Feb 2015.

34. Fox K, Ford I, Steg PG, Tendera M, Robertson M, Ferrari R. Relationship between ivabradine treatment and cardiovascular outcomes in patients with stable coronary artery disease and left ventricular systolic dysfunction with limiting angina: a subgroup analysis of the randomized, controlled BEAUTIFUL trial. Eur Heart J. 2009;30(19):2337-45.

35. Fox K, Ford I, Steg PG, Tardif JC, Tendera M. Ferrari $\mathrm{R}$; SIGNIFY Investigators. Ivabradine in stable coronary artery disease without clinical heart failure. N Engl J Med. 2014;371(12):1091-9.

36. Baptista S. POST-IT (Portuguese Study on The Evaluation of FFR Guided Treatment of Coronary 
Disease) registry. In: Presented at Congress of the European Association of Percutaneous Cardiovascular Interventions, Paris, France. 2014.

37. De Bruyne B, Fearon WF, Pijls NH, Barbato E, Tonino P, Piroth Z, the FAME 2 Trial Investigators, et al. Fractional flow reserve-guided PCI for stable coronary artery disease. $\mathrm{N}$ Engl J Med. 2014;371(13):1208-17.

38. Bristow MR, Saxon LA, Boehmer J, Krueger S, Kass DA, De Marco T, et al. Cardiac-resynchronization therapy with or without an implantable defibrillator in advanced chronic heart failure. N Engl J Med. 2004;350:2140-50.

39. Cleland JG, Daubert JC, Erdmann E, Freemantle N, Gras D, Kappenberger L, et al. The effect of cardiac resynchronization on morbidity and mortality in heart failure. N Engl J Med. 2005;352:1539-49.

40. Bisch L, Da Costa A, Dauphinot V, RomeyerBouchard C, Khris L, M'Baye A, et al. Predictive factors of difficult implantation procedure in cardiac resynchronization therapy. Europace. 2010;12:1141-8.

41. Cazeau S, Leclercq C, Lavergne T, Walker S, Varma C, Linde C, et al. Effects of multisite biventricular pacing in patients with heart failure and intraventricular conduction delay. $\mathrm{N}$ Engl J Med. 2001;344:873-80.

42. Boriani G. Cardiac resynchronization therapy with a novel quadripolar lead decreases complications at six months: preliminary results of the MORE-CRT trial. In: Presented at European Society of Cardiology, August 31, Barcelona, Spain. 2014.

43. St. Jude Medical products. The Leadless Pacemaker. http://www.sjm.com/leadlesspacing/intl/options/ leadless-pacing. Accessed 28 Feb 2015.
44. Reddy VY, Knops RE, Sperzel J, Miller MA, Petru J, Simon J, et al. Permanent leadless cardiac pacing: results of the LEADLESS trial. Circulation. 2014;129(14):1466-71.

45. Martinez C, Katholing A, Freedman SB. Adverse prognosis of incidentally detected ambulatory atrial fibrillation. A cohort study. Thromb Haemost. 2014;112(2):276-86.

46. Anguera Camos I. Efficacy of quinidine in patients with Brugada syndrome and frequent ventricular arrhythmia episodes. In: Presented at European Society of Cardiology, August 31, Barcelona, Spain. 2014.

47. Cannon C. IMPROVE-IT: Examining Outcomes in Subjects With Acute Coronary Syndrome: Vytorin (Ezetimibe/Simvastatin) vs Simvastatin (P04103). In: Presented at American Heart Association, November 18, Chicago, Illinois, USA. 2014.

48. Moriarty PM, Jacobson TA, Bruckert E, Thompson PD, Guyton JR, Baccara-Dinet MT, Gipe D. Efficacy and safety of alirocumab, a monoclonal antibody to PCSK9, in statin-intolerant patients: design and rationale of ODYSSEY ALTERNATIVE, a randomized phase 3 trial. J Clin Lipidol. 2014;8(6):554-61.

49. Muhlestein JB, Lappé DL, Lima JA, Rosen BD, May HT, Knight S, Bluemke DA, Towner SR, Le V, Bair TL, Vavere AL, Anderson JL. Effect of screening for coronary artery disease using CT angiography on mortality and cardiac events in high-risk patients with diabetes: the FACTOR-64 Randomized Clinical Trial. JAMA. 2014;. doi:10.1001/jama.2014.15825. 